\section{Horses for courses}

The number of patients receiving treatment for chronic renal failure by dialysis and transplantation continues to increase: there are now about 64000 patients on regular dialysis throughout the world. Nevertheless, the number of patients treated per million of the population varies considerably from country to country. Britain ranks seventeenth in a world league table, treating 62 patients per million, compared with the league leader, Switzerland, whose rate is 136 . The European Dialysis and Transplant Association Congress, held in Hamburg in June, was told that there had been a recent substantial increase in the number of patients treated as a result of a change in the criteria for acceptance on renal replacement programmes. The average age of patients starting dialysis in Europe has increased from 36 in 1970 to 40 in 1975 and in the USA there has been a similar rise from 39 to 45 years over the same period. Furthermore, patients with systemic disease leading to end stage renal failure are no longer being excluded from dialysis programmes. The most common of these is diabetes mellitus, and in Europe in the past 12 months there has been an $82 \%$ increase in diabetics starting treatment; indeed, diabetics accounted for $3.5 \%$ of all new patients receiving dialysis in 1975. There has also been a $50 \%$ increase in patients with systemic lupus erythematosus and a $47_{\%}^{\circ}$ increase in patients with amyloidosis.

With so many patients being treated by various methods we may now compare the cumulative survival rates. For only those patients between 14 and 34 years of age, survival on hospital dialysis was $83 \%$ at three years in Britain, compared with a European average of $65 \%$. Home dialysis, the most widely used form of dialysis here, has a survival rate approaching $82 \%$ at three years. As might be expected, cumulative patient survival for patients with a systemic disease is less than that for patients with isolated kidney failure. For example, it is roughly $65 \%$ at two years for systemic lupus erythematosus and only $50 \%$ tt two years for patients with amyloidosis and diabetes. After cadaveric transplantation diabetics have a less than $25 \%$ graft survival at two years compared with the European average of just less than $40 \%$ for other patients at three years. Data presented at the meeting from Australia and New Zealand (where renal transplantation is considered the primary method of treatment) showed a slightly better graft survival, being $47 \%$ at five years.

We now have enough information for a rational choice of treatment for any individual patient. For example, in patients over 44 years of age graft survival with cadaveric transplantation is less than $40 \%$ and continues to decrease with increasing age. This decreased survival rate is attributable to nonimmunological causes such as infection and cardiovascular mishapsa fact emphasised by several large series of transplant results presented from centres throughout Europe and Scandinavia. Factors such as previous blood transfusion and the order of transplantation do not appear to affect graft survival adversely, though presensitisation does. Conflicting data were presented on graft survival in patients who were or had been hepatitis $B$ antigen (HBAg) positive, and, though there was no adverse effect on graft survival in such patients in the short term, about $10 \%$ of deaths in long-term survivors were due to hepatic failure. Renal units in Britain can be justifiably pleased that there have been no new cases of hepatitis or the presence of positive HBAg in their nursing staff in the last two years. We should note the continuing high incidence among the staff of European centres that dialyse or transplant positive patients.
Also of note was the $8 \%$ incidence of malignant tumours in the Australian transplant series, rising to almost $25 \%$ in the 5-10 year survivors. Many of these were skin cancers (common in Australasia) and could be treated accordingly, but others, such as tumours of lymphatic tissue, often proved fatal.

Everyone concerned with long-term dialysis is familiar with the difficulties of adequate fluid removal during haemodialysis. Rapid ultrafiltration in the fluid-overloaded patient often results in hypotension, nausea, vomiting, and muscular cramps. Bergström and his colleagues from Sweden, however, claimed that ultrafiltration alone (without dialysis) could remove up to 3 litres of fluid in three hours from patients without a significant change in blood pressure, pulse rate, or plasma osmolarity, despite a $20 \%$ decrease in plasma volume; and the patients felt no adverse effect. The mechanism by which plasma volume could be decreased by $20 \%$ without apparent changes in blood pressure and pulse is, as yet, ill understood, but it was postulated that rapid changes in plasma osmolarity might interfere with regulation of blood pressure. This technique is likely to prove of great value in patients with acute renal failure, where fluid overload and hypotension often occur. Fluid has also been removed by ultrafiltration in other states of excess fluid retention, such as congestive cardiac failure, and a modified form of ultrafiltration treatment has also been tried in a group of patients with -chronic renal failure for up to 12 months. While the blood urea and serum creatinine concentrations were higher in these patients than those on standard dialysis treatment there was a comparable improvement in the patients' general health. Hypertension has been easily controlled, and nerve conduction velocity also increased in all patients. This latter finding was attributed to the increased removal of middle molecules (molecular weight 1200 to 1500 ) during ultrafiltration. The full potential of this simple and cheap technique will become apparent only with its wider use and a better understanding of the physiological mechanisms, especially in acutely ill patients.

\section{Metabolic bone disease in Asians}

The health problems of the estimated 900000 Asians living in Britain are largely similar to those of the rest of the population, including other immigrant groups. Asians differ strikingly, however, in their propensity to develop vitamin D deficiency. Rickets and oestomalacia in Indian and Pakistani immigrants were first reported in Glasgow in 1962 and have since been found in almost every centre of immigrant population. ${ }^{2-5}$

In White children and in the children of other immigrant groups infantile rickets is found in urban families in poor social circumstances who do not avail themselves of vitamin Dfortified welfare foods or vitamin D supplements. ${ }^{6}$ Rickets in children over 3 years old should lead to a search for malabsorption or renal disease. In contrast, in Asian children active rickets occurs not only in infancy but throughout school life, with a peak incidence at puberty, and children of all socioeconomic groups are affected. Adults may develop osteomalacia, the adult counterpart of rickets, especially during or after pregnancy, ${ }^{7}$ and a vitamin D-deficient mother may give birth to a child with fetal rickets. ${ }^{8}$ Though common 
in Asia, these manifestations of vitamin $\mathrm{D}$ deficiency have not been seen in Britain for many years, and unfamiliarity with their clinical presentation may delay diagnosis.

The paediatrician may encounter Asian rickets as hypocalcaemic tetany which does not respond to the administration of calcium supplements or in the infant of an Asian mother who does not use welfare foods. The general practitioner or school doctor may encounter it in an adolescent who complains of painful legs when walking, running, or doing gymnastics; physical examination may show the genu valgum characteristic of "knock-kneed" late rickets in severe cases. The obstetrician may meet osteomalacia in a patient with thoracic, back, and limb pains, who has bony tenderness over the rib cage and elsewhere; the general physician or general practitioner may encounter a similar clinical picture in Asian patients of any age.

The condition is often missed at an early stage-as reports of osteotomies for rachitic deformity in Asian children testify. ${ }^{3}$ Once considered, the diagnosis is easily confirmed by finding low serum concentrations of calcium or inorganic phosphate. combined with a raised alkaline phosphatase. In severe cases radiological evidence of rachitic epiphyses and the typical pseudofractures of osteomalacia may be found combined with bony deformity. Treatment is simple: patients respond readily to $2-3000$ units of vitamin $\mathrm{D}$ daily or to a single intramuscular injection of 600000 units.

The reasons for the prevalence of Asian rickets are not clear. The dietary vitamin $\mathrm{D}$ intakes of many children with Asian rickets are similar to those of White or West Indian children, who are unaffected beyond infancy. ${ }^{9-11}$ Ultraviolet deprivation has been suggested, ${ }^{12}{ }^{13}$ but measurements of the outdoor exposure of Asian children have shown no differences from White controls. ${ }^{14}$ The consumption of high-extraction chapati flour by most Asians has been blamed, ${ }^{15}{ }^{16}$ but the mechanism by which chapati flour might produce vitamin $\mathrm{D}$ deficiency remains obscure. Phytic acid, ${ }^{1516}$ phytate-derived inositol polyphosphates, ${ }^{17}$ dietary phosphorus, ${ }^{14}$ or malabsorption of vitamin $D$ induced by a diet high in phytate and vegetable fibre $^{18}$ have all been suggested. Finally, genetic differences in intermediary vitamin $\mathrm{D}$ metabolism may be partly responsible. ${ }^{719}$

Patients with Asian rickets show low serum concentrations of 25-hydroxy-vitamin $\mathrm{D},{ }^{20}$ and 400 units of vitamin $\mathrm{D}$ daily will restore these to normal, ${ }^{21}$ providing effective prophylaxis. The problem for the preventive health services is to ensure that the population at risk gets this simple prophylactic measure. Clinical cases of rickets and osteomalacia are the tip of an iceberg of hypovitaminosis D-which is effectively the whole Asian population. The record of the last 14 years shows that exhortations to Asians to consume more vitamin D-containing foods such as oily fish or margarine are unlikely to be effective, and more vigorous action is needed. Welfare services for preschool children are in general well organised, but there is no evidence of any concerted action on the part of school health services to provide vitamin D supplements for Asian schoolchildren: 3000 units of vitamin D weekly would be sufficient. The adult population is more difficult to reach, but much more could be done by community health teams to distribute information on the need for vitamin $\mathrm{D}$ supplements to Asian families through Indian and Pakistani social and cultural associations.

The simplest way to eliminate vitamin $\mathrm{D}$ deficiency from the Asian community is to fortify a foodstuff consumed exclusively by most of the population at risk. Vitamin D-fortified chapati flour will raise levels of serum 25-hydroxy-vitamin $\mathrm{D}$ to normal and heal the rickets of those consuming it. ${ }^{21}$
Fortification is technically straightforward, and the additional cost could be minimal. The introduction of this method of prophylaxis should be considered urgently by the Committee on Medical Aspects of Food Policy. A study in Bradford ${ }^{21}$ has indicated that as many as 1 in 40 Asian children may expect admission to hospital with rickets before 16 years. Many Asian children have rachitic deformities. These are distressing reminders of a failure of the preventive medicine services. Further delay in introducing effective prophylactic measures is difficult to defend.

${ }^{1}$ Dunnigan, M G, ct al, Scottish Medical fournal, 1962, 7, 159.

2 Swan, C H J, and Cooke, W T, Lancet, 1971, 2, 456.

${ }^{3}$ Ford, J A, et al, British Medical fournal, 1972, 2, 677

${ }^{4}$ Holmes, A M, et al, Quarterly fournal of Medicine, 1973, 42, 125.

${ }^{5}$ Moncrieff, M W, Lunt, H R W, and Arthur, L J H, Archives of Disease in Childhood, 1973, 48, 221.

6 Benson, P F, et al, British Medical fournal, 1963, 1, 1054

' Felton, D J C, and Stone, W D, British Medical fournal, 1966, 1, 1521.

${ }^{8}$ Ford, J A, et al, British Medical fournal, 1973, 3, 211.

9 Dunnigan, M G, and Smith, C M, Scottish Medical fournal, 1965, 10, 1.

10 Watney, P J M, et al, British Medical fournal, 1971, 2, 432.

11 Cooke, W T, et al, British Medical fournal, 1974, 2, 293.

12 Hodgkin, P, et al, Lancet, 1973, 2, 168.

13 Gupta, M M, Round, J M, and Stamp, T C B, Lancet, 1974, 1, 586.

${ }^{14}$ Dunnigan, M G, et al, Scottish Medical fournal, 1975, 20, 217.

15 Wills, M R, et al, Lancet, 1972, 1, 771.

${ }^{16}$ Ford, J A, et al, British Medical fournal, 1972, 3, 446

17 Van den Berg, C J, Hill, L F, and Stanbury, S W, Clinical Science, 1972, 43, 377.

${ }^{18}$ Polanska, N, Dale, R A, and Wills, M R, Annals of Clinical Biochemistry, 1976, 13, 339.

19 Doxiadis, S, et al, Archives of Disease in Childhood, 1976, 51, 83.

${ }^{20}$ Preece, M A, et al, Lancet, 1973, 1, 907.

${ }_{21}$ Pietrek, J, et al, Lancet, 1976, 1, 1145.

\section{Replacement of the knee joint}

Replacement of the knee joint is still at an early stage of development, unlike the operation on the hip that has now become routine. Yet the demand equals that for the hip, and since arthrodesis of the knee leads to severe disability ${ }^{1}$ the need may be as great if not greater.

There are four indications for replacement of any jointpain, instability, stiffness, and deformity. In the case of the knee pain and instability are the most important. Stiffness alone would not be accepted as grounds for operation by most surgeons unless it was associated with stiffness of other joints and these contributed to severe incapacity, and deformity would warrant surgery only if it was associated with instability or pain.

A suitable prosthesis must relieve pain, simulate the normal joint movements, provide stability, and correct deformity. In addition arthrodesis should be possible if the prosthesis has to be removed later. The hinge joints used in the $1950 \mathrm{~s}$ and early 1960s had disadvantages in that they allowed movement around a fixed axis only and therefore tended either to break or to become loose. Their relatively large size meant that so much bone had to be excised at insertion that in the event of a failure arthrodesis was difficult or impossible. The two-piece prostheses which came next were smaller and depended on the patient's own ligaments for stability, but they were not suitable when gross joint destruction or instability was present. A recent advance has been the concept of a two-piece prosthesis with a mechanical link to give stability. Such a joint allows the gliding movements of flexion and extension with some rotation and lateral mobility, as in the normal knee. ${ }^{2}$ The mechanical link acts in place of the 